\title{
Tuning of Surface-Acoustic-Wave Dispersion via Magnetically Modulated Contact Resonances
}

\author{
Antonio Palermo, ${ }^{*}$ Yifan Wang, Paolo Celli, and Chiara Daraio \\ Division of Engineering and Applied Science, California Institute of Technology, Pasadena, California 91125,
} USA

(Received 6 January 2019; revised manuscript received 20 March 2019; published 18 April 2019)

\begin{abstract}
In this work, we propose the use of contact resonances, controlled via an external magnetic field, as a tunable platform to manipulate the dispersion of surface acoustic waves (SAWs). We exploit the analogy between surface acoustic waves in a semi-infinite medium and edge waves in a plate, to realize a compact experimental setup and to demonstrate our tuning strategy. The setup consists of a set of ferromagnetic bead resonators in contact with thin, permanent magnets and positioned at the free edge of an elastic plate. An additional set of magnets, placed at a controlled and variable distance from the beads, is used to alter the contact stiffness and natural frequencies of the bead resonators. We exploit resonances to open large-frequency band gaps via edge-wave hybridization and implement our tuning strategy to shift their frequency ranges. We predict the tuned dispersive properties of hybridized edge waves via numerical models and experimentally reconstruct them via laser vibrometry, finding excellent agreement. The use of magnetic interaction and contact mechanics as a tuning strategy for SAW systems could pave the way toward programmable devices for sensing and electronic components.
\end{abstract}

DOI: 10.1103/PhysRevApplied.11.044057

\section{INTRODUCTION}

Controlling the propagation of surface acoustic waves (SAWs) can provide unique opportunities to design devices across vastly different length scales, ranging from compact radio-frequency components in wireless telecommunication systems [1] at the micro- and nanoscale, to wave barriers and seismic cloaks [2-5] at the geophysical scale. These applications require the design of structured surface layers, e.g., phononic crystals and metamaterials, that can interact with SAWs and control their dispersive properties. As an example, periodic patterns of microscopic holes on an elastic substrate can be used to design filters for SAWs [6] and waveguides [7], exploiting Bragg scattering phenomena. Similarly, ordered and disordered arrays of surface pillars are able to induce subwavelength band gaps due to the hybridization between the SAW modes and pillars' resonances [8]. These hybrid modes can be exploited for subwavelength waveguiding $[9,10]$ and to achieve negative lensing effects [11]. The same hybridization phenomenon is observed when SAWs interact with a granular layer of silica microbeads, owing to the contact resonance of each sphere adhered to the elastic substrate. These granular metamaterials have been thoroughly investigated with the goal of understanding the microbeads'

\footnotetext{
*antonio.palermo6@unibo.it
}

contact dynamics $[12,13]$ and to realize SAW filters in the megahertz and gigahertz range [14].

Granular systems present an additional desirable feature with respect to other platforms - the possibility of tuning their response by altering the contact stiffness. The tunability of contact mechanics via external precompression has been explored at the mesoscale for multiple engineering applications, e.g., filters [15-17], acoustic lenses [18], and rectifiers [19]. More recently, external magnetic fields have been used to control the dynamics of ferromagnetic beads for the realization of one-dimensional tunable magnetogranular chains, where the interparticle contact forces are varied by changing the applied field [20,21]. The prospect of achieving tunability by using external, noncontact interaction forces is also particularly appealing in the context of SAWs, where examples of tunable systems are still limited [22,23] with respect to bulk and plate structures [24-29]. In light of these considerations, in this work, we propose and demonstrate a strategy for SAW manipulation that involves contact-based ferromagnetic resonators controlled via an external magnetic field. Our tabletop experimental setup consists of a thin plate, serving as a two-dimensional (2D) model, equipped with an array of ferromagnetic resonators on the plate edge. The compact setup is used to experimentally measure the tuning of plate edge waves, whose dispersive properties are analogous to those of SAWs in a semi-infinite medium $[30,31]$. We tune the resonances of the ferromagnetic beads 
by changing the position of a second array of permanent magnets, thus altering the frequency ranges over which hybridization band gaps occur. While we use this platform to demonstrate band-gap shifting, the same paradigm could be seamlessly extended to other wave manipulation effects, such as subwavelength waveguiding or lensing [32].

\section{A "COMPACT" SAW EXPERIMENTAL SETUP}

Our experimental setup is shown in Fig. 1(a). It consists of a $610 \times 927 \times 9 \mathrm{~mm}(H \times L \times t)$ acrylic plate (PMMA) with Young's modulus $E=5.5 \mathrm{GPa}$, Poisson's ratio $v=$ 0.35 , and density $\rho=1190 \mathrm{~kg} \mathrm{~m}^{-3}$. The plate is clamped to an optical table at its bottom edge and is decorated with an array of 44 permanent magnets (K\&J magnetics B662-N52) $((\mathrm{Nd}, \mathrm{Fe}) \mathrm{B})$ at its top edge. The magnets have dimensions of $9 \times 9 \times 3 \mathrm{~mm}\left(a_{m} \times a_{m} \times t_{m}\right)$ and a relative spacing $s=15 \mathrm{~mm}$ [see Fig. 1(b)]. A steel bead (McMaster-Carr 9642K49) with radius $r_{b}=4.75 \mathrm{~mm}$ and mass $M=3.5 \mathrm{~g}$ is placed atop each magnet to realize an array of mechanical resonators. An identical array of magnets is arranged on a movable acrylic beam hanging over the bead chain at a prescribed distance $d$, variable within the range $0-25 \mathrm{~mm}$. Vertically polarized plate edge waves are excited using an acoustic coil (HiWave HIAX11C00532), placed at a distance $l_{m}=130 \mathrm{~mm}$ from the first bead, and driven by a signal generator (Agilent 33220A) and an audio amplifier (Topping TP30). The signal of choice is a wide-band Ricker wavelet centered at $10 \mathrm{kHz}$. For this wavelet, the characteristic wavelength of the traveling wave on the pristine elastic plate, i.e., $\lambda \approx 120 \mathrm{~mm}$, is significantly larger than the bead dimensions and spacing. A laser doppler vibrometer (LDV, Polytec OFV-5000) mounted on a motorized linear stage is used to measure the vertical velocity component of desired points along the plate's top edge. The data are acquired using an oscilloscope (Tektronix DPO3034) and postprocessed in Matlab. The use of thin plates to probe the dynamic of SAWs in semi-infinte media is supported by the similarity between Rayleigh waves and extensional waves traveling at the edge of a semi-infinite plate, which share an analogous dispersive relationship. For the sake of clarity, we here report the analytical dispersion relation of extensional waves traveling on the edge of a semi-infinite elastic plate. The interested reader can find the detailed derivation in Refs. $[30,33]$. For a thin plate $(t \ll \lambda)$ occupying the $x-z$ plane from $z=0$ to $z=-\infty$, with free stress boundary conditions at $z=0$, the phase velocity $c$ of edge waves traveling in the $x$ direction can be calculated from

$$
\left(2-\frac{c^{2}}{c_{T}^{2}}\right)^{2}-4\left(1-\frac{c^{2}}{c_{T}^{2}}\right)^{1 / 2}\left(1-\frac{c^{2}}{c_{P, \mathrm{pl}}^{2}}\right)^{1 / 2}=0,
$$

where $c_{P, \mathrm{pl}}=\left[E / \rho\left(1-v^{2}\right)\right]^{1 / 2}$ is the velocity of dilatational waves in the plate. Note that Eq. (1) is identical to
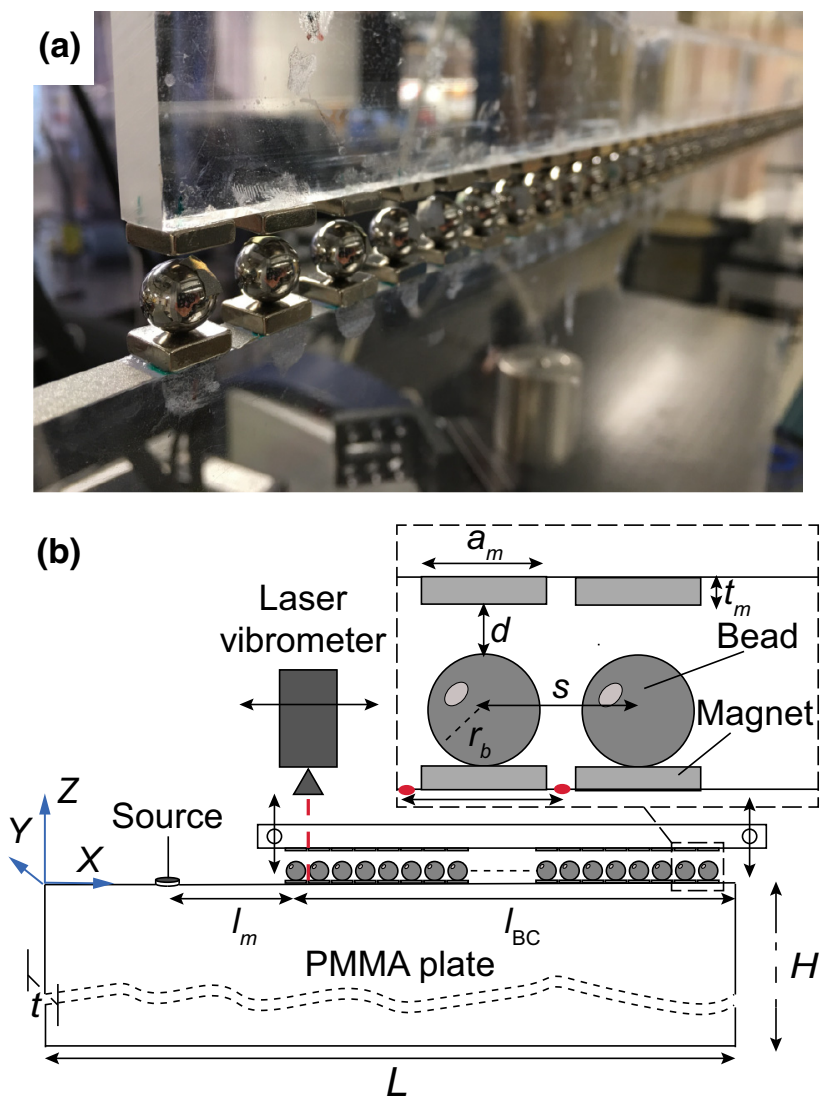

FIG. 1. (a) Picture of the experimental setup and (b) detailed schematic of it, indicating all the components and geometrical parameters of interest.

the one describing Rayleigh waves in a semi-infinite solid [34], with the plate dilatational velocity replacing the bulk dilatational velocity. Indeed, the use of thin sheets and edge modes to discuss the dynamics of SAWs is a standard in the context of seismology [30,31], where 2D models are preferred for the ease of fabrication and lower setup cost.

\section{SAW-CONTACT-RESONANCE INTERACTION}

To predict and interpret the results of our experimental platform, we analyze the dynamics of an array of resonant beads in contact with an elastic substrate. We first recall the dynamics of a resonant bead attached to a rigid substrate, resorting to the model discussed in Ref. [35]. We utilize this analytical model to derive the linearized response of the bead resonance and to obtain a discrete description (i.e., a two-degree-of-freedom (DOF) model) of the bead dynamics. Then, we utilize finite-element simulations to discuss the bead-substrate interaction, employing the discrete bead description to model the resonator response.

\section{A. Dynamics of a single resonant bead}

We model the bead as a rigid body of mass $M$, with the sphere-substrate contact represented by normal and shear 
springs [Fig. 2(a)]. The normal spring is described via Hertz's contact model [36]; thus, the force-displacement law between an elastic sphere and an elastic half-space reads

$$
F_{c}=\frac{4}{3} E^{*} r_{b}^{1 / 2} \delta^{3 / 2},
$$

with $E^{*}=\left[\left(1-v_{b}^{2}\right) / E_{b}+\left(1-v_{m}^{2}\right) / E_{m}\right]^{-1}$, where $\delta$ is the normal contact displacement, and where $E_{b}, E_{m}, v_{b}$, and $v_{m}$ are the Young's moduli and Poisson's ratios of the bead $(b)$ and magnet $(\mathrm{m})$, respectively. The Hertzian interaction can be linearized about a static displacement $\delta_{0}$, leading to the expression of the normal stiffness:

$$
K_{N}=2 E^{*} r_{b}^{1 / 2} \delta_{0}^{1 / 2}
$$

Similarly, the shear spring $K_{S}$ is derived from Mindlin's shear contact force-displacement law [37]:

$$
F_{s}=8 G^{*} r_{b}^{1 / 2} \delta_{s} \delta^{1 / 2}
$$

with $G^{*}=\left[\left(2-v_{b}\right) / G_{b}+\left(2-v_{m}\right) / G_{m}\right]^{-1}$, where $G_{b}$ and $G_{m}$ are the shear moduli of bead and magnet, respectively, and $\delta_{s}$ the displacement of the bead center of mass in the direction transverse to the contact normal. By linearizing
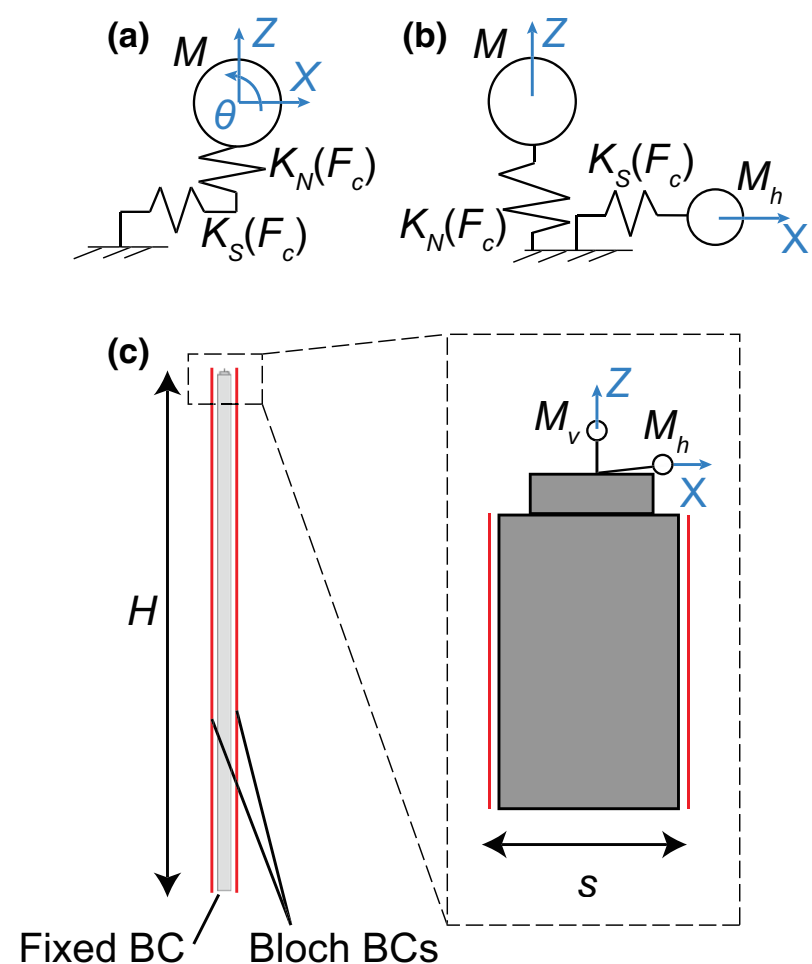

FIG. 2. Schematic of a single bead over a rigid substrate: (a) three-DOF and (b) equivalent two-DOF system. (c) Schematic of the wave-finite-element model.
Eq. (4) about the equilibrium position $\delta_{0}, \delta_{s 0}$, and setting $\delta_{s 0}=0$, the shear spring constant $K_{S}$ is

$$
K_{S}=8 G^{*} r_{b}^{1 / 2} \delta_{0}^{1 / 2}=4 \frac{G^{*}}{E^{*}} K_{N} .
$$

Equipped with a linearized description of the contact stiffness, we describe the dynamics of a single bead using a three-DOF system, namely the horizontal $(X)$ and vertical $(Z)$ displacement of the bead's centroid and the bead rotation $(\theta)$ with respect to its central axis of inertia, following the approach detailed in Ref. [35]. Assuming small displacements about the equilibrium position $\left(\delta_{0}, \delta_{s 0}\right)$, the free vibrations of this system can be described by the following set of equations:

$$
\begin{aligned}
M \ddot{Z}+K_{N} Z & =0, \\
M \ddot{X}+K_{S}\left(X+r_{b} \theta\right) & =0, \\
I \ddot{\theta}+K_{S} r_{b}\left(r_{b} \theta+X\right) & =0,
\end{aligned}
$$

where $I=\frac{2}{5} M r_{b}^{2}$ is the moment of inertia of the sphere. As evident from Eq. (6), the system is characterized by an uncoupled vertical mode with a resonance frequency $f_{v}=\sqrt{K_{N} / M} / 2 \pi$. In addition, two coupled horizontalrotational motions exist, one of them being a rigid rototranslation, i.e., $f_{\mathrm{HR}}=0$, and the second one with a resonance at $f_{\mathrm{RH}}=\sqrt{7 K_{S} /(2 M)} / 2 \pi$ (see details on the isolated sphere dynamics in Ref. [35]). Hence, by discarding the rigid motion, we can represent the bead dynamics by a set of two orthogonal resonators, with spring constants $K_{N}$ and $K_{S}$, respectively, and mass $M_{v}=M$ and $M_{h}=\frac{2}{7} M$ [Fig. 2(b)]. In the following, we adopt this set of orthogonal resonators to couple the dynamics of a contact resonance with SAWs traveling in the elastic substrate.

\section{B. Resonant beads on an elastic substrate}

We now analyze the dispersive properties of an infinite array of bead-magnet resonators resting on an elastic substrate. The coupling between SAWs and randomly distributed surface oscillators was first analyzed in Ref. [38]. Closed-form dispersion relations for uniformly distributed resonators were then derived in Refs. [12,35] utilizing an effective medium approach. Although efficient, this approach remains valid only in the longwavelength regime, where the dispersive effects induced by the periodicity of the beads' array are negligible. In our experiments, because of the additional presence of periodically distributed permanent magnets, such effects cannot be disregarded, preventing us from utilizing such closed-form analytical solutions. For this reason, we resort to finite-element simulations to model an infinite array of magnet-bead units over an elastic substrate, employing a wave-finite-element (WFE) approach. Specifically, we consider a 2D FE model of the system's unit cell in 
COMSOL Multiphysics and restrict our analysis to vertically polarized surface waves. The unit cell comprises a vertical strip of plate of width equal to the magnet spacing $(s)$, a magnet, and the bead-contact resonator modeled by a couple of orthogonal mass-spring resonators to simulate both vertical and horizontal resonances as detailed in Secs. III A [Fig. 2(c)]. We assume plane-stress conditions. To model each mass-spring resonator, we utilize a truss element with a point mass at the truss tip [39,40]. To account for periodicity, we impose Bloch-Floquet boundary conditions (BCs) on the vertical edges of the cell, along the direction of wave propagation. Fixed boundary conditions are applied at the bottom of the unit cell to simulate the boundary conditions of the setup. The elastic substrate and the magnet domains are discretized with a converged mesh of quadratic triangular elements, ensuring a minimum number of six elements for the shortest wavelength considered. The contact springs are modeled with linear Lagrangian truss elements, sufficient to describe the stiffness of the single-DOF resonators. Dispersion curves are calculated setting up and solving an eigenfrequency problem for 50 wavenumber values within the range $k=1 / \lambda=0-1 /(2 s) 1 / \mathrm{m}$.

\section{RESULTS}

\section{A. Magnetic force and contact-resonance tuning}

We now consider the tuning effects provided by the movable array of permanent magnets introduced in Sec. II. To characterize this tuning effect, we measure the pulling force required to position the bead at varying distances $d=0-10 \mathrm{~mm}$ from the magnet, utilizing a standard testing machine (Instron E3000), as shown in Fig. 3(a). The measured pulling force is fitted using the interpolation function

$$
F_{m}(d)=\frac{p_{0}}{p_{d 2} d^{2}+p_{d 1} d+p_{d 0}}
$$

$\left(p_{0}=1 \mathrm{~N}, p_{d 2}=0.07414 \mathrm{~m}^{-2}, p_{d 1}=0.03975 \mathrm{~m}^{-1}, p_{d 0}=\right.$ 0.1739), which is characteristic of a dipole interaction and adequately describes the measured force (Fig. 3(b)). We then utilize the expression in Eq. (7) to estimate the net contact force $F_{c}(d)$ exerted by a bead over the surface magnet $m_{1}$ when a second magnet $m_{2}$ (the one providing the tuning) is placed at a distance $d_{2}=d$ from the sphere [as in Fig. 4(a)], as follows:

$$
F_{c}(d)=F_{m_{1}}-F_{m_{2}}=F_{m}(0)-F_{m}(d),
$$

where $F_{m_{1}}$ and $F_{m_{2}}$ are the forces exerted by the two magnets on the bead. Using this contact force prediction, we can calculate the bead's vertical resonance frequency $f_{v}(d)$ by means of Eqs. (2), (3), and (6). First, we calculate the bead's vertical resonance without magnet $m_{2}$, (a)

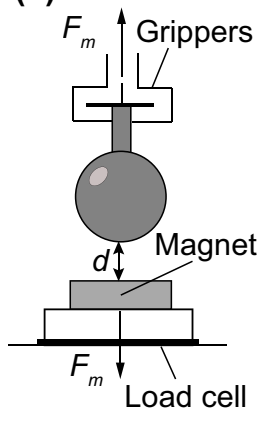

(b)

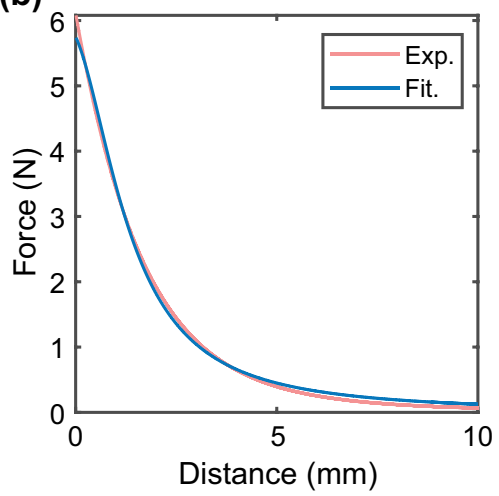

FIG. 3. Experimental characterization of the bead-magnet interaction force. (a) Schematic of the experimental setup. (b) Results, featuring experimental and fitted data.

i.e., when $F_{c}=F_{m 1}=5.7 \mathrm{~N}$. For our steel bead $\left(E_{b}=\right.$ $210 \mathrm{GPa}, v_{b}=0.3$ [41]) in contact with the surface of a nickel-coated magnet $\left(E_{m}=190 \mathrm{GPa}, v_{m}=0.3\right.$ [41]), this amounts to $f_{v}=\sqrt{K_{N} / M}=9.5 \mathrm{kHz}$. The same model predicts a variation of the resonance frequency in the range $f_{v}(d)=5.4-9.4 \mathrm{kHz}$ for a distance $d=0.2-5.0 \mathrm{~mm}$ [blue line in Fig. 4(b)]. Given the proportionality between the vertical and horizontal linear spring constants in Eq. (5), the same frequency tunability can be achieved for the horizontal mode, which can be varied in the range $f_{\mathrm{RH}}(d)=$ 9.2-16 kHz (see the Appendix).

We verify these analytical predictions using the experimental setup illustrated in Fig. 4(b). The bead, in contact with the permanent magnet $m_{1}$, is excited using a longitudinal piezoelectric transducer (Panametrics V1011). On the other side, a second magnet $m_{2}$ is positioned at a given distance $d=0.1270-5.080 \pm 0.125 \mathrm{~mm}$ to replicate the conditions assumed in the analytical model. The bead is excited using a Ricker pulse centered at $10 \mathrm{kHz}$ and its transient response is recorded using our LDV. For each distance $d$, we perform three measurements and evaluate the average resonance frequency $f_{v}(d)$. The experimental resonances [markers with error bars in Fig. 4(b)] show excellent agreement with the analytical predictions, confirming our ability to largely tune the bead's dynamics. As a representative example, Figs. 4(c) and 4(d) provide the time $(t)$ and frequency $(f)$ normalized amplitude (Norm. Ampl.) response, i.e. $A_{t, f} / \max \left(A_{t, f}\right)$, of the bead for a distance $d=1.01 \pm 0.125 \mathrm{~mm}$; in this specific case, the bead resonates at $8.35 \mathrm{kHz}$ with a quality factor $Q \approx 10$.

\section{B. SAW dispersion tuning}

We experimentally investigate the interaction between surface waves and arrays of resonators. We record the vertical velocity wave field over an ensemble of 41 
(a)
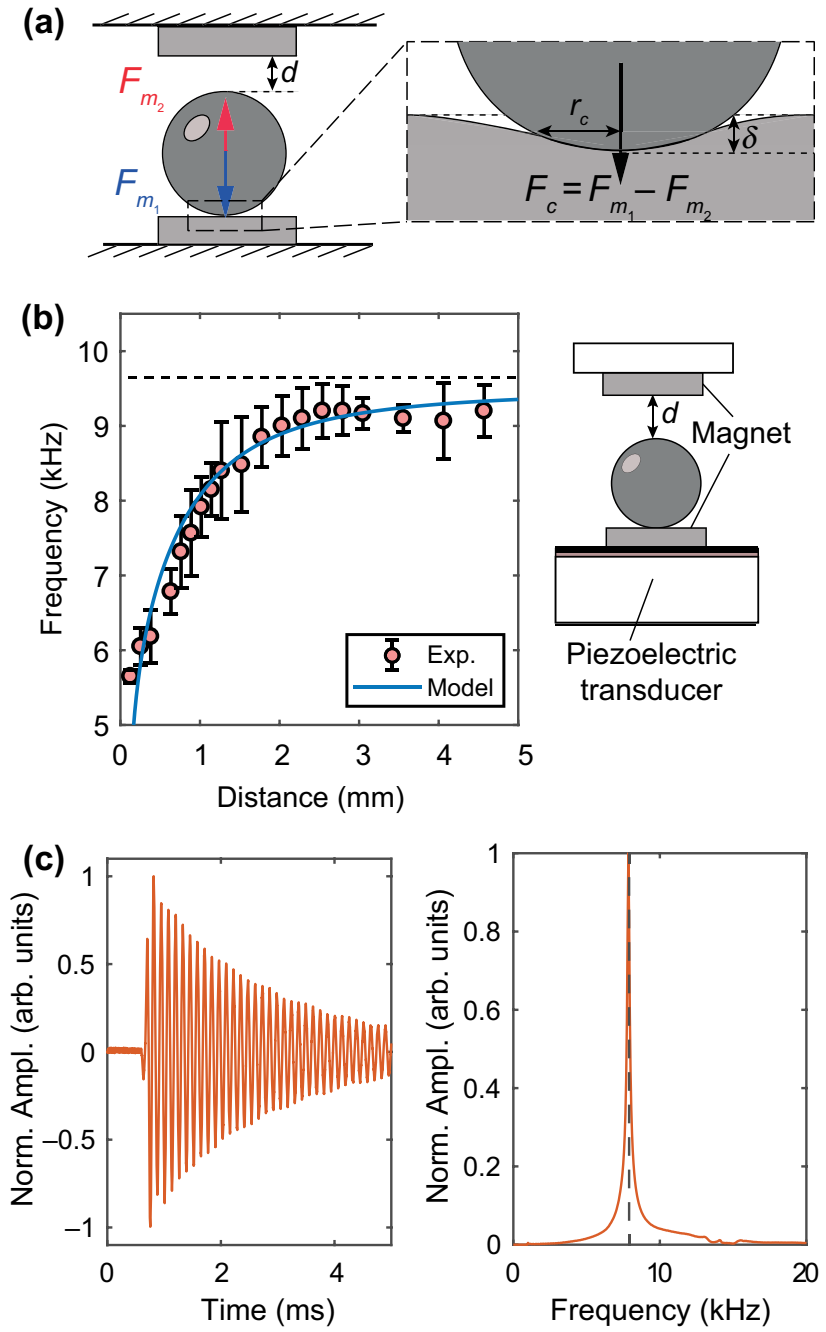

FIG. 4. (a) Illustration of the contact-force tuning. (b) Experimental and numerical characterization of the bead vertical resonance tuning. Examples of a measured (c) time and (d) frequency velocity response of a magnet-bead resonator tuned with a permanent magnet $\left(m_{2}\right)$ placed at a distance $d=1.01 \pm 0.125 \mathrm{~mm}$. Norm. Ampl., normalized amplitude.

measurement points located between magnets. All measurements are carried out using a small-amplitude excitation, to verify the linearized bead dynamics discussed in Sec. III. We record the surface velocity time histories and postprocess the data via 2D Fourier transforms, to reconstruct the experimental dispersion curves (Fig. 5). We reconstruct the dispersion of the pristine plate, the plate with magnets and no beads, and the plate with magnet-bead resonators (untuned). These measurements are conducted to (i) validate experimentally the possibility of investigating SAWs in a compact, platelike setup; (ii) understand the dispersive features introduced by the presence of magnets on the surface; and (iii) highlight the fundamental dynamics of SAWs interacting with contact resonances and validate the numerical WFE model.
The experimental dispersion curve for the pristine plate is shown in Fig. 5(a). The Ricker pulse generated by the acoustic coil travels as a nondispersive mode with a velocity $c_{R}=1205 \mathrm{~m} \mathrm{~s}^{-1}$, which can be predicted analytically using the Rayleigh-like dispersion relation in Eq. (1), marked in the plot by the dashed red line. Note that, although the Ricker pulse is centered at $10 \mathrm{kHz}$, we observe a significant amplification of the signal content within the range $12-15 \mathrm{kHz}$, possibly related to the dynamics of the acoustic coil. Placing magnets on the plate's surface introduces a dispersive character to the fundamental SAW, which "bends" toward the Bragg limit $\left(k_{\text {Bragg }}=33.33 \mathrm{~m}^{-1}\right)$ and deviates from the sound cone region $\left(f / k \geq c_{T}\right.$, marked by the dashed black line) due to the periodicity of the array [Fig. 5(b)]. This behavior is well captured by the WFE predictions (dashed red line) and provides the baseline to interpret the additional dispersive features introduced by the collective beads' resonances. The addition of surface resonators manifests in the dispersion curve as a hybridization between the traveling wave and the collective resonance modes. In our experiment, the vertical $\left(f_{v}\right)$ and rototranslational $\left(f_{\mathrm{RH}}\right)$ resonances of the beads interact with the dispersive SAW, resulting in two slow-propagating flat branches [Fig. 5(c)], similarly to what has been observed in microscale dynamics [35]. Note that the frequencies where these modes flatten mark the beads' resonances. With respect to our single-bead predictions, we here observe a significant shift of the bead vertical (from 9.5 to $8.1 \mathrm{kHz}$ ) and rototranslational resonance (from 16 to $12.2 \mathrm{kHz}$ ), which we ascribe to the coupling between the rigid contact dynamics and the softer elastic substrate. These frequency shifts are naturally accounted for in the WFE model, which accurately predicts the dynamics of such hybrid modes. The hybridization phenomenon is accompanied by an "avoided crossing" behavior, which results in a SAW band gap, where energy is leaked into the substrate bulk [12]. In detail, each frequency gap is bounded between a resonance frequency and the intersection of the upper dispersive branch with the bulk shear wave line, i.e., $\Delta f_{i}=\left[f_{i, \mathrm{BG}^{-}}-\right.$ $\left.f_{i, \mathrm{BG}^{+}}\right]$, with $f_{i, \mathrm{BG}^{-}}=f_{i}$ and $f_{i, \mathrm{BG}^{+}}=k c_{T}$, where $i$ is the band-gap number (either 1 or 2). In our setup, according to the numerical predictions, this condition translates into a negligible lower frequency gap $\Delta f_{1}=8.2-8.3 \mathrm{kHz}$ and into a second wider gap $\Delta f_{2}=12.2-16.0 \mathrm{kHz}$. Numerical predictions are confirmed by the experimental dispersion curve: at $f=14 \mathrm{kHz}$, a representative frequency inside the band gap, the system with beads shows a significantly lower amplitude $A \approx 0.2 A_{\max }$ [Fig. 5(c)] with respect to the case without beads, where $A \approx A_{\max }$ [Fig. 5(a)].The reader can find examples of recorded time histories used to extract the dispersion curve in Figs. 5(a) and 5(c) of the Appendix. To demonstrate the tunability of our platform, we analyze the dispersive behavior of SAWs interacting with modulated contact resonances, 
(a)

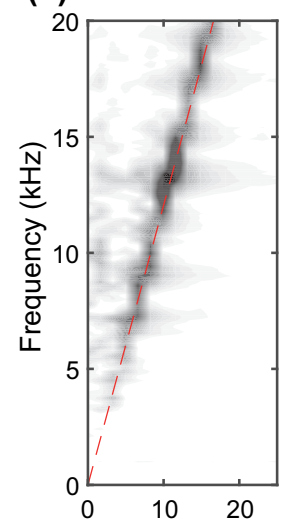

(b)

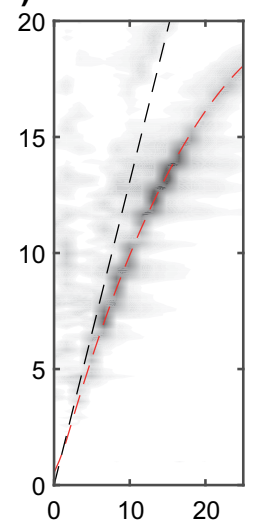

(c)

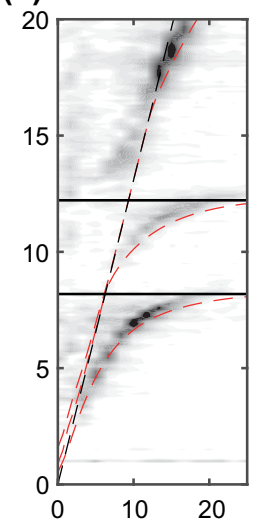

(d) $d=1.5 \mathrm{~mm}$

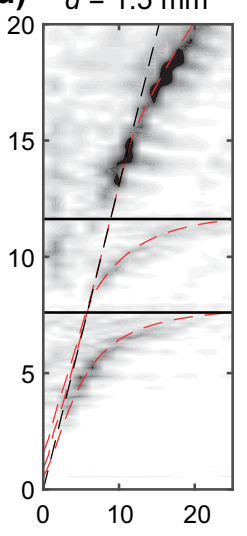

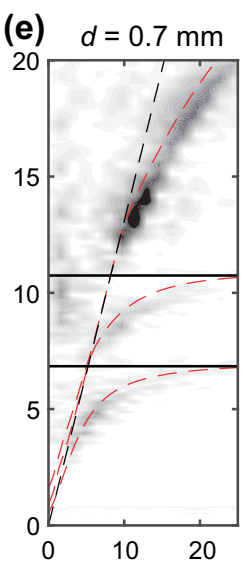

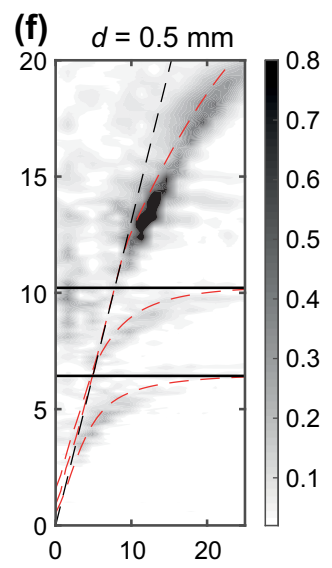

Wavenumber $(1 / \mathrm{m})$ Wavenumber $(1 / \mathrm{m})$ Wavenumber $(1 / \mathrm{m})$ Wavenumber $(1 / \mathrm{m})$ Wavenumber $(1 / \mathrm{m})$ Wavenumber $(1 / \mathrm{m})$

FIG. 5. Experimental characterization of SAWs interacting with contact resonances, with dispersion curves of (a) SAWs traveling in a pristine plate; (b) SAWs traveling in the plate decorated with the array of magnets; (c) SAWs interacting with the array of magnet-bead resonators; and SAWs interacting with the array of magnet-bead resonators modulated with a second chain of magnets placed at a distance (d) $d=1.5 \mathrm{~mm}$, (e) $d=0.7 \mathrm{~mm}$, (f) $d=0.5 \mathrm{~mm}$. Gray color maps are obtained as 2D Fourier transforms of the experimental time-space data. The red dashed curves are the dispersion branches predicted by the WFE model [except for panel (a), where the prediction is obtained analytically, according to Eq. (1)], while the gray dashed one marks the shear nondispersive branch. The horizontal gray lines indicate the analytical resonances and coincide with the gaps' onsets.

obtained by positioning an array of hanging magnets at a decreasing distance $d=[1.5,0.7,0.5] \pm 0.2 \mathrm{~mm}$ from the bead. As expected, the large tunability of contact resonances discussed in Secs. IV A results in the possibility of significantly changing the position of the hybridized branches and, in turn, the position and width of the related SAW band gaps. For example, for a modulating distance $d=1.5 \mathrm{~mm}$, experimental and numerical results predict two hybrid modes approaching, respectively, the tuned resonances $f_{v}(1.5 \mathrm{~mm})=7.5 \mathrm{kHz}$ and $f_{\mathrm{RH}}(1.5 \mathrm{~mm})=11.1$ $\mathrm{kHz}$; they also highlight an upper SAW band gap extending in the $\Delta f_{2}(1.5 \mathrm{~mm})=11.6-13.7 \mathrm{kHz}$ range [Fig. $5(\mathrm{~d})$ ]. It is worth pointing out that the numerical model, updated using the tuned contact force formula in Eq. (8), does not require any additional fitting parameter to predict the measured frequency shifts. As expected, larger resonance and band-gap variations are achieved for smaller modulating distances $d$, i.e., $\Delta f_{2}(0.7 \mathrm{~mm})=10.7-12.5 \mathrm{kHz}$, $\Delta f_{2}(0.5 \mathrm{~mm})=10.2-11.7 \mathrm{kHz}$ [Figs. 5(e) and 5(f)]. For these configurations, at the representative frequency $f=$ $14 \mathrm{kHz}$, which now lies outside the tuned band gaps, no amplitude attenuation is observed [Figs. 5(d)-5(f)]. Note that, for very short bead-magnet distances $(d=0.5-0.7$ $\mathrm{mm}$ ), the energy traces of the lower hybrid branches are less pronounced, due to the lower amount of energy convened by the Ricker excitation to frequencies below 8 $\mathrm{kHz}$ and to the finite precision of our positioning system, which causes a slight inherent variability between resonances of different beads in the array. Removing this variability, e.g., via automatically controlled position systems, would allow us to also achieve a larger range of tunability.

\section{CONCLUSIONS}

In this work, we demonstrate how SAWs can be attenuated at desired frequencies using an array of tunable contact resonances. We show that mechanical resonators can be easily realized with spherical beads in contact with permanent magnets. By exploiting the large sensitivity to contact forces of the beads and modulating the contact forces using an additional movable array of magnets, we demonstrate the possibility of tuning the bead resonances. This result, in turn, allows us to control the hybridization gaps arising from the coupling between traveling waves and bead resonances and, hence, filter out the propagation of edge modes at selected frequency ranges. Although the proposed strategy has been discussed by means of a 2D experimental setup, our results can be seamlessly extended to the dynamic of SAWs traveling in a homogeneous half-space, provided that: (i) the plate dilatational velocity is substituted by the medium bulk dilatational velocity [30] and (ii) the one-dimensional (1D) array of beads is extended to a 2D surface array. In this regard, one should employ a number of surface resonators per unit area $n_{A}=A /(s \times t)$, to ensure analogous results, i.e., bandgap width and shift, to those of a plate with thickness $t(y$ direction) and resonator spacing $s$ ( $x$ direction), for surface waves propagating in the $x$ direction. Minor changes are introduced along the other directions due to the different unit-cell periodicity. In addition, we believe that a similar tuning paradigm could be implemented at the microscale and nanoscale, where contact-based metamaterials made of micro- and nanobeads deposited on a silica substrate have already been utilized to filter SAWs in the rf regime 
[12-14]. Within this context, resonance tuning via a magnetic field could be achieved by employing, for example, magnetic microbeads, similar to those already commercialized for magnetic separation techniques [42], and modulating their contact resonances via an external magnetic field.

\section{ACKNOWLEDGMENTS}

A.P. acknowledges the support of the California Institute of Technology through the Cecil and Sally Drinkward Postdoctoral Fellowship. Y.W. and C.D. acknowledge the support from the National Science Foundation under EFRI Grant No. 1741565. We thank Luca Bonanomi for fruitful discussions.

\section{APPENDIX A: ADDITIONAL EXPERIMENTAL DATA}

\section{Tuning of the bead horizontal resonance}

We calculate and experimentally measure the horizontal resonance frequency $f_{\mathrm{RH}}(d)$ of a bead in contact with a permanent magnet $m_{1}$ and modulated by a second magnet $m_{2}$ placed at a distance $d$ from the bead surface. We utilize Eqs. (2), (5), and (6), to predict a variation of the resonance frequency between $f_{\mathrm{RH}}(d)=10.8-16 \mathrm{kHz}$ for a distance $d=0.2-5.0 \mathrm{~mm}$ [blue line in Fig. 6(b)]. To measure the horizontal resonance frequency, we utilize the experimental setup illustrated in Fig. 6(a). We provide an excitation orthogonal to the normal contact at the base of the beadmagnet assembly using a longitudinal piezoelectric transducer (Panametrics V1011). Again, a second magnet $m_{2}$ is positioned at selected distances from the bead's surface to replicate the conditions assumed in the analytical model. We excite the bead using a Ricker pulse centered at $10 \mathrm{kHz}$. We record its lateral response with our LDV and perform three measurements to evaluate its average resonance frequency $f_{\mathrm{RH}}(d)$. The experimental resonances, marked with

(a)

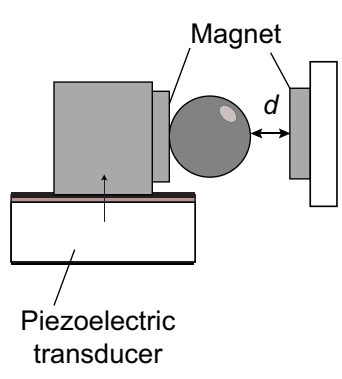

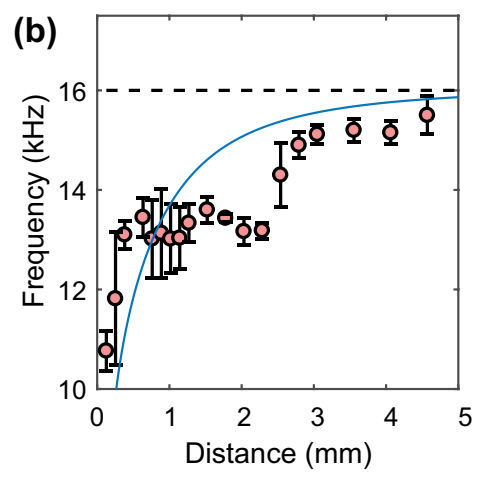

FIG. 6. Characterization of the bead's horizontal resonance. (a) Schematic of the experimental setup. (b) Experimental and numerical results.
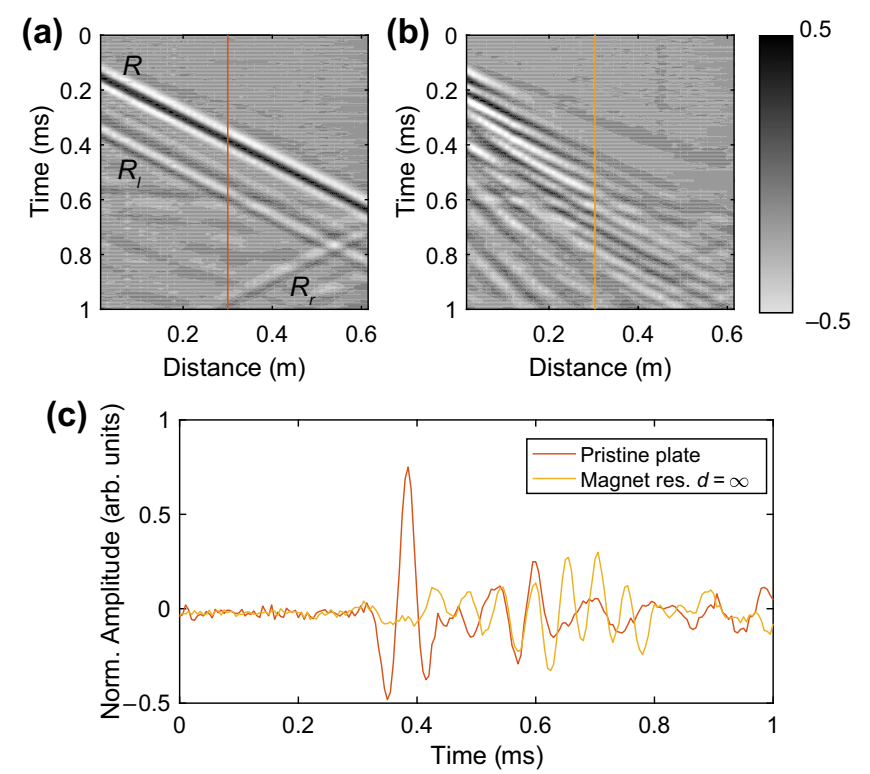

FIG. 7. Examples of LDV velocity measurements for a Ricker pulse traveling in (a) a pristine plate and (b) a plate equipped with magnet-bead resonators (and no modulating force, i.e., $d=$ $\infty$ ). (c) Time histories of the measured velocity responses at a position $x=30 \mathrm{~cm}$.

error bars in Fig. 6(b), are in good agreement with the analytical predictions for small and large tuning distances. We observe some discrepancy at distances between 1 and 2 $\mathrm{mm}$, possibly related to additional frictional effects or to some lateral confinement induced by the magnets and not accounted for in the model.

\section{Time-history records}

Example of time-history records, used to obtain the disperive properties of the pristine plate and of the plate equipped with magnet-bead resonators [Figs. 5(a) and 5(c)], are displayed in Fig. 7. These time histories confirm that the Ricker wavelet generated by the acoustic coil propagates as a compact, i.e., nondispersive, edge mode in the pristine plate [see signal (R) in Fig. 7(a)]. This feature is followed by lower-amplitude reflected signals $\left(R_{l}, R_{r}\right)$, generated at the plate's left $(l)$ and right $(r)$ boundaries. Conversely, the presence of the resonant beads spreads the arrival of the wave packet at different times, highlighting the dispersive features introduced by the resonances [Fig. 7(b)]. The signal dispersion can be further appreciated by comparing the velocity traces extracted at a distance $x=30 \mathrm{~cm}$ for the pristine plate and plate with resonant beads [Fig. 7(c)].

[1] J. D. Maines and E. G. S. Paige, Surface-acoustic-wave devices for signal processing applications, Proc. IEEE 64, 639 (1976). 
[2] M. Miniaci, A. Krushynska, F. Bosia, and N. M. Pugno, Large scale mechanical metamaterials as seismic shields, New J. Phys. 18, 083041 (2016).

[3] A. Palermo, S. Krödel, A. Marzani, and C. Daraio, Engineered metabarrier as shield from seismic surface waves, Sci. Rep. 6, 39356 (2016).

[4] A. Diatta, Y. Achaoui, S. Brûlé, S. Enoch, and S. Guenneau, Control of Rayleigh-like waves in thick plate Willis metamaterials, AIP Adv. 6, 121707 (2016).

[5] S. R. Sklan, R. Y. Pak, and B. Li, Seismic invisibility: Elastic wave cloaking via symmetrized transformation media, New J. Phys. 20, 063013 (2018).

[6] S. Benchabane, A. Khelif, J. Y. Rauch, L. Robert, and V. Laude, Evidence for complete surface wave band gap in a piezoelectric phononic crystal, Phys. Rev. E - Stat. Nonlinear, Soft Matter Phys. 73, 065601 (2006).

[7] O. B. Wright and O. Matsuda, Watching surface waves in phononic crystals, Philos. Trans. R. Soc. A: Math. Phys. Eng. Sci. 373, 20140364 (2015).

[8] Y. Achaoui, A. Khelif, S. Benchabane, L. Robert, and V. Laude, Experimental observation of locally-resonant and Bragg band gaps for surface guided waves in a phononic crystal of pillars, Phys. Rev. B - Condens. Matter Mater. Phys. 83, 104201 (2011).

[9] M. Addouche, M. A. Al-Lethawe, A. Elayouch, and A. Khelif, Subwavelength waveguiding of surface phonons in pillars-based phononic crystal, AIP Adv. 4, 124303 (2014).

[10] M. Al Lethawe, M. Addouche, S. Benchabane, V. Laude, and A. Khelif, Guidance of surface elastic waves along a linear chain of pillars, AIP Adv. 6, 121708 (2016).

[11] M. Addouche, M. A. Al-Lethawe, A. Choujaa, and A. Khelif, Superlensing effect for surface acoustic waves in a pillar-based phononic crystal with negative refractive index, Appl. Phys. Lett. 105, 023501 (2014).

[12] N. Boechler, J. K. Eliason, A. Kumar, A. A. Maznev, K. A. Nelson, and N. Fang, Interaction of a Contact Resonance of Microspheres with Surface Acoustic Waves, Phys. Rev. Lett. 111, 036103 (2013).

[13] M. Hiraiwa, M. Abi Ghanem, S. P. Wallen, A. Khanolkar, A. A. Maznev, and N. Boechler, Complex Contact-Based Dynamics of Microsphere Monolayers Revealed by Resonant Attenuation of Surface Acoustic Waves, Phys. Rev. Lett. 116, 198001 (2016).

[14] P. H. Otsuka, S. Mezil, O. Matsuda, M. Tomoda, A. A. Maznev, T. Gan, N. Fang, N. Boechler, V. E. Gusev, and O. B. Wright, Time-domain imaging of gigahertz surface waves on an acoustic metamaterial, New J. Phys. 20, 013026 (2018).

[15] N. Boechler, J. Yang, G. Theocharis, P. G. Kevrekidis, and C. Daraio, Tunable vibrational band gaps inonedimensional diatomic granular crystals with three-particle unit cells, J. Appl. Phys. 109, 074906 (2011).

[16] L. Bonanomi, G. Theocharis, and C. Daraio, Wave propagation in granular chains with local resonances, Phys. Rev. E - Stat. Nonlinear Soft Matter Phys. 91, 033208 (2015).

[17] E. B. Herbold, J. Kim, V. F. Nesterenko, S. Y. Wang, and C. Daraio, Pulse propagation in a linear and nonlinear diatomic periodic chain: Effects of acoustic frequency band-gap, Acta Mech. 205, 85 (2009).
[18] A. Spadoni and C. Daraio, Generation and control of sound bullets with a nonlinear acoustic lens, Proc. Natl. Acad. Sci. 107, 7230 (2010).

[19] N. Boechler, G. Theocharis, and C. Daraio, Bifurcationbased acoustic switching and rectification, Nat. Mater. 10, 665 (2011).

[20] F. Allein, V. Tournat, V. E. Gusev, and G. Theocharis, Tunable magneto-granular phononic crystals, Appl. Phys. Lett. 108, 16 (2016).

[21] F. Allein, V. Tournat, V. E. Gusev, and G. Theocharis, Transversal-rotational and zero group velocity modes in tunable magneto-granular phononic crystals, Extreme Mech. Lett. 12, 65 (2017).

[22] N. Cselyuszka, M. Seçujski, N. Engheta, and V. CrnojevićBengin, Temperature-controlled acoustic surface waves, New J. Phys. 18, 103006 (2016).

[23] S. M. Yuan, T. X. Ma, A. L. Chen, and Y. S. Wang, Liquidassisted tunable metasurface for simultaneous manipulation of surface elastic and acoustic waves, AIP Adv. 8, 035026 (2018).

[24] O. Bou Matar, J. F. Robillard, J. O. Vasseur, A. C. HladkyHennion, P. A. Deymier, P. Pernod, and V. Preobrazhensky, Band gap tunability of magneto-elastic phononic crystal, J. Appl. Phys. 111, 054901 (2012).

[25] F. Casadei, T. Delpero, A. Bergamini, P. Ermanni, and M. Ruzzene, Piezoelectric resonator arrays for tunable acoustic waveguides and metamaterials, J. Appl. Phys. 112, 064902 (2012).

[26] A. Bergamini, T. Delpero, L. D. Simoni, L. D. Lillo, M. Ruzzene, and P. Ermanni, Phononic crystal with adaptive connectivity, Adv. Mater. 26, 1343 (2014).

[27] P. Wang, F. Casadei, S. Shan, J. C. Weaver, and K. Bertoldi, Harnessing Buckling to Design Tunable Locally Resonant Acoustic Metamaterials, Phys. Rev. Lett. 113, 014301 (2014).

[28] Z. Wang, Q. Zhang, K. Zhang, and G. Hu, Tunable digital metamaterial for broadband vibration isolation at low frequency, Adv. Mater. 28, 9857 (2016).

[29] O. R. Bilal, A. Foehr, and C. Daraio, Reprogrammable phononic metasurfaces, Adv. Mater. 29, 1700628 (2017).

[30] J. Oliver, F. Press, and M. Ewing, Two-dimensional model seismology, Geophysics 19, 202 (1954).

[31] T. T. Goforth, Model study of the effect on the rayleigh spectrum of lateral heterogeneity in earthquake source regions, J. Geophys. Res. 81, 3599 (1976).

[32] A. Palermo and A. Marzani, Control of Love waves by resonant metasurfaces, Sci. Rep. 8, 7234 (2018).

[33] M. V. Wilde, M. V. Golub, and A. A. Eremin, Experimental and theoretical investigation of transient edge waves excited by a piezoelectric transducer bonded to the edge of a thick elastic plate, J. Sound Vib. 441, 26 (2019).

[34] K. F. Graff, Wave Motion in Elastic Solids (Dover, New York, 1975), p. 688.

[35] S. P. Wallen, A. A. Maznev, and N. Boechler, Dynamics of a monolayer of microspheres on an elastic substrate, Phys. Rev. B - Condens. Matter Mater. Phys. 92, 174303 (2015).

[36] K. L. Johnson, Contact Mechanics (Cambridge University Press, Cambridge, 1985).

[37] R. D. Mindlin, Compliance of elastic bodies in contact, J. Appl. Mech. 16, 259 (1949). 
[38] A. P. Mayer, Interaction of rayleigh waves with randomly distributed oscillators on the surface, Phys. Rev. B - Condens. Matter Mater. Phys. 59, 13291 (1999).

[39] A. Palermo, M. Vitali, and A. Marzani, Metabarriers with multi-mass locally resonating units for broad band Rayleigh waves attenuation, Soil Dyn. Earthquake Eng. 113, 265 (2018).

[40] A. Palermo, S. Krödel, K. H. Matlack, R. Zaccherini, V. K. Dertimanis, E. N. Chatzi, A. Marzani, and C. Daraio,
Hybridization of Guided Surface Acoustic Modes in Unconsolidated Granular Media by a Resonant Metasurface, Phys. Rev. Appl. 9, 054026 (2018).

[41] M. F. Ashby, Materials Selection in Mechanical Design, 5th edition (Butterworth-Heinemann, Oxford, 2018), p. 660.

[42] J. Tao, Z. Liu, Q. Lu, Z. Ding, X. Chen, X. Wang, G. $\mathrm{Du}, \mathrm{K}$. Yao, and F. Gong, Preparation and characterization of polymer-coated core-shell structured magnetic microbeads, J. Magn. Magn. Mater. 265, 98 (2003). 